\title{
NEMO-binding domain peptide ameliorates inflammatory bone destruction in a Staphylococcus aureus-induced chronic osteomyelitis model
}

\author{
Yanhua Lan, YuWang ${ }^{*}$,Zhijian Xie* \\ Affiliated Hospital of Stomatology, Medical College, Zhejiang University, Hangzhou, \\ Zhejiang 310006,China
}

Figure 1
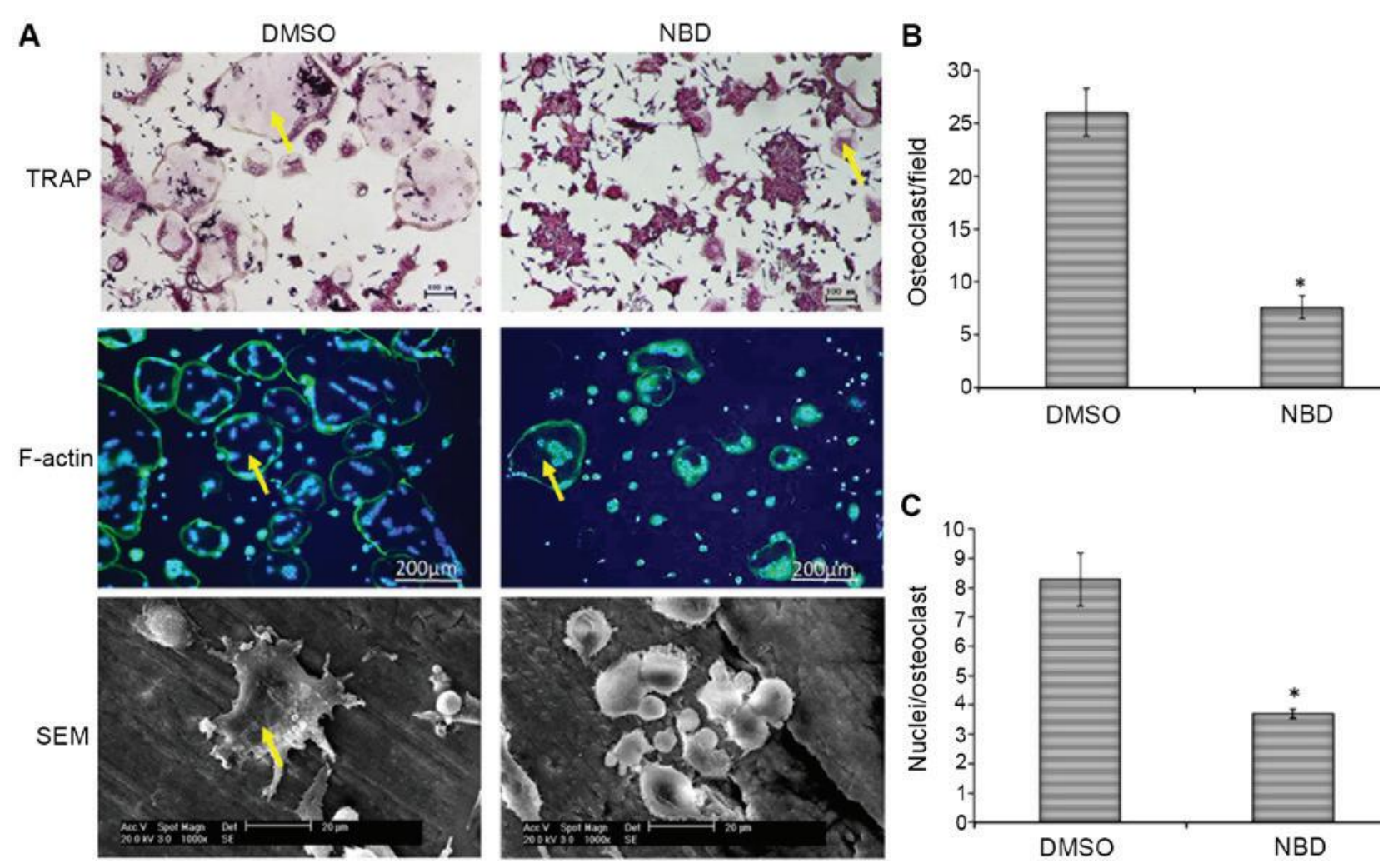

Figure 1. NBD peptide inhibits RANKL-induced osteoclast formation function in vitro.

\section{Backgroud}

Osteomyelitis, which is characterized by progressive inflammatory bone destruction and resorption, is a difficultto-treat infection.Staphylococcus aureus (S. aureus) is one of the major causes of this disease. A cell-permeable peptide inhibitor of the IKB kinase complex, the nuclear factor (NF). $\kappa \mathrm{B}$ essential modulator-binding domain (NBD) peptide, has been reported to block osteoclastogenesis and may be considered a potential strategy for preventing inflammatory bone resorption.

\section{Methods:}

In order to investigate the role of NBD in S. aureus-inducedc steomyelitis, the present study

obtained the NBD peptide, and the function and formation of Osteoclast were observed in vitro. Subsequently, a bone defect was generated and S. aureus was injected into the mandible of experimental animals, in order to establish an in vivo osteomyelitis model. The present study analyzed the following three experimental groups:

Untreated, treated with debridement, and treated with debridement plus NBD peptide administration.

\section{Results:}

The results suggested that the NBD peptide reduced RANKL-induced osteoclast formation and function in vitro.
Figure 2

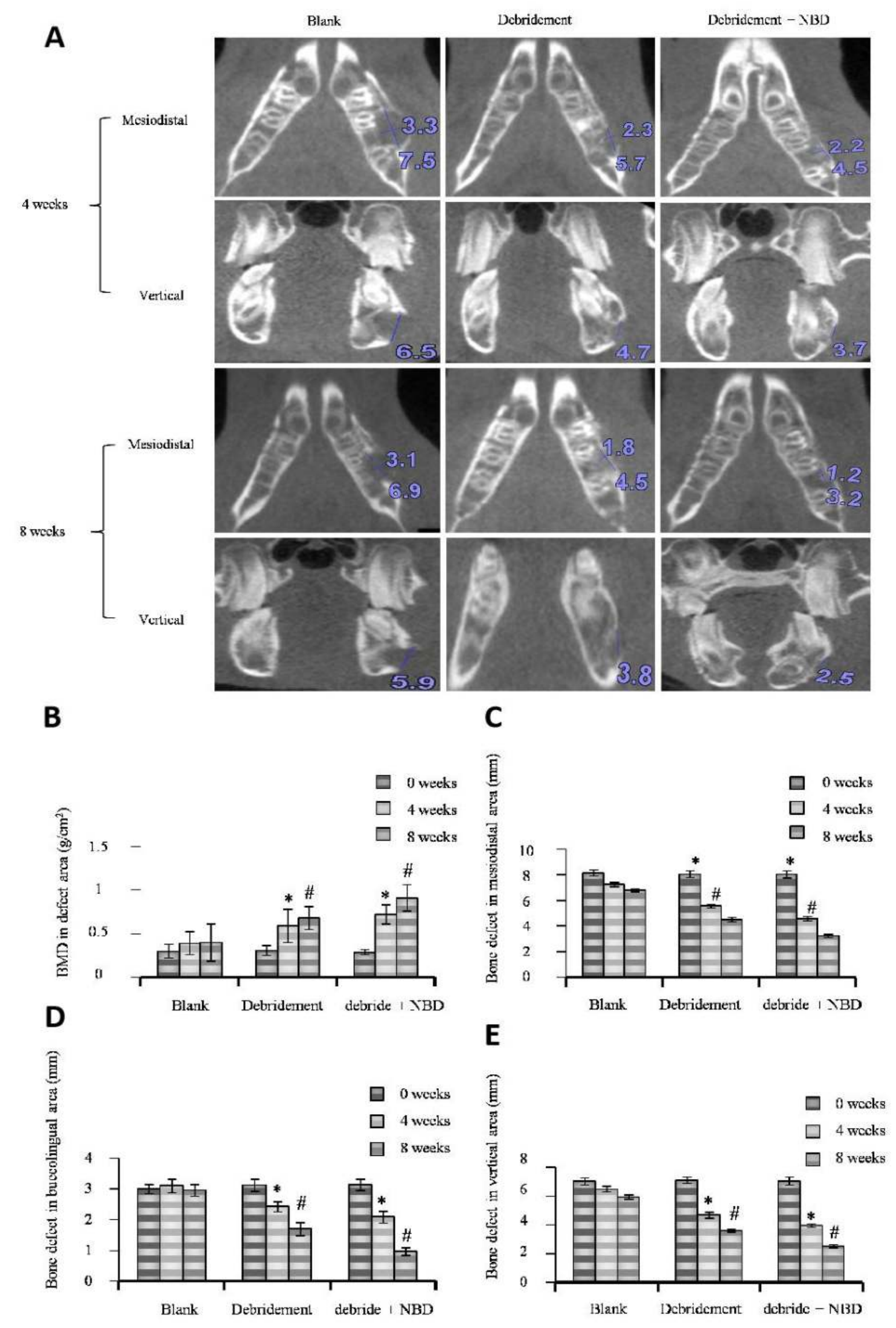

Figure 2. (A) NBD peptide ameliorates bone resorption in an in vivo model of mandibular osteomyelitis, as shown by CBCT. (B-D) NBD reduces bone defects in mandibular osteomyelitis. BMD, bone mineral density; NBD, nuclear factor- $\kappa B$ essential modulator-binding domain.

And the results in vivo model of osteomyelitis demonstrated that treatment with the NBD peptide reduced the bone defect in a 3-dimensional manner, and reduced bone resorption.

\section{Conclusions:}

To the best of our knowledge, the present study is the first to demonstrate that, in a model of osteomyelitis caused by S. aureus, the NBD peptide serves a role in inhibiting osteolysis and promoting bone remodeling in the direction of osteogenesis. The effects were better than those produced by debridement alone, thus suggesting that it may have promising therap eutic potential in osteomyelitis. 